\title{
Diplomacia de negócios e relações públicas internacionais
}

\author{
Business diplomacy and international public relations
}

Diplomacia de negocios y relaciones públicas internacionales

\section{Guilherme Fráguas Nobre}

- Pós-doutor pela Université Stendhal, França

- Pós-doutorando da Escola de Comunicações e Artes da Universidade de São Paulo (ECA-USP)

- Doutor em Ciências da Comunicação pela ECA-USP

- Mestre em Desenvolvimento Econômico pela Universidade Federal do Paraná (UFPR)

- Graduado em Ciências Econômicas pela Universidade Federal de Minas Gerais (UFMG)

- Afiliação à Universidade de São Paulo e à Universitat de Girona - Catalunha, Espanha

- Visiting Scholar na McMaster University, Canadá

• E-mail: fraguasnobre@gmail.com; fraguas@usp.br

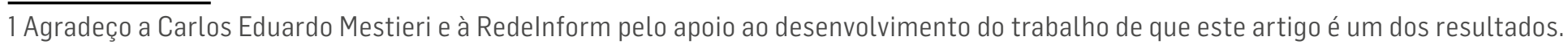




\section{Resumo}

Recentemente tem-se assistido à democratização da diplomacia, isto é, à ampliação do acesso e da participação de múltiplos stakeholders nos negócios internacionais - públicos e privados. Este artigo vai analisar o que aproxima duas áreas erroneamente consideradas distintas, como a diplomacia e as relações públicas - ou, para ser mais exato, a diplomacia de negócios e as relações públicas internacionais. Ambas envolvem a indicação de representantes, trabalham essencialmente na esfera dos relacionamentos sociais e atuam concomitantemente na esfera local e global. As relações públicas internacionais têm muito a ganhar com este paralelo, uma vez que poderiam ser pensadas em larga medida como um tipo de "diplomacia civil". Embora não seja acurado defender a perfeita sinonímia entre diplomacia de negócios e relações públicas internacionais, é, sim, cabível afirmar que ambas comungam mais afinidades do que diferenças.

\section{PALAVRAS-CHAVE: DIPLOMACIA DE NEGÓCIO • RELACÕ̃ES PÚBLICAS INTERNACIONAIS・DIPLOMACIA・RELACÕ̃ES PÚBLICAS.}

\section{Abstract}

Recently one has observed the democratization of diplomacy, i.e., the broadening of the access and of the participation of multiple stakeholders in international business - both public and private. This article will analyze what brings closer two areas that are erroneously considered to be distinct, such as diplomacy and public relations - or, to be more precise, the business diplomacy and the international public relations. Both involve the designation of representatives, work essentially in the sphere of social relationships and act concomitantly in the local and global spheres. International public relations have much to gain from this parallel, inasmuch as they could be to a large extent seen as a kind of "civil diplomacy". Although it may not be absolutely correct to defend the perfect synonymy between business diplomacy and international public relations, it is perfectly pertinent to affirm that both of them share in common more affinities than differences.

\section{KEYWORDS: BUSINESS DIPLOMACY •INTERNATIONAL PUBLIC RELATION・DIPLOMACY・PUBLIC RELATIONS.}

\section{Resumen}

Recientemente se ha asistido a la democratización de la diplomacia, es decir, asistimos a la ampliación del acceso y de la participación de múltiplos stakeholders en los negocios internacionales - públicos y privados. Este artículo va a analizar lo que aproxima dos áreas erróneamente consideradas distintas, como la diplomacia y las relaciones públicas; 0, para ser más exacto, la diplomacia de negocios y las relaciones públicas internacionales. Ambas envuelven la indicación de representantes, trabajan esencialmente en la esfera de los relacionamientos sociales y actúan concomitantemente en la esfera local y global. Las relaciones públicas internacionales tienen mucho para ganar con este paralelo, una vez que podrían ser pensadas en gran medida como un tipo de "diplomacia civil". Aunque no sea adecuado defender la perfecta sinonimia entre diplomacia de negocios y relaciones públicas internacionales, es posible afirmar sí que ambas comulgan más afinidades que diferencias. 
á quem defina um diplomata como um especialista em generalidades, em parte porque dele se exige grande flexibilidade e elevado grau de adaptação às mais diversas (e dinâmicas) situações e interlocutores. Algumas das atividades mais corriqueiras desempenhadas pelo pessoal diplomático envolvem, também, marcar passagens e hotéis, recepcionar e ciceronear pessoas durante sua estadia na cidade, acompanhá-las em compras e restaurantes, apresentá-las a artistas e autoridades, organizar e comparecer a eventos, enfim, uma série de ocupações que podem estar isentas do glamourgeralmente associado à profissão.

Alguns profissionais de relações públicas, em geral, e de relações públicas internacionais, em particular, vão, porventura, se identificar com isso. Tal se dá porque as relações públicas e as relações públicas internacionais sofrem, às vezes, com a imperfeita nitidez que parte da sociedade capta em sua profissão, e com certa desvalorização do mercado acerca do correto cumprimento de um rol imenso de atividades que estão na base e no cerne do sucesso da atividade principal de vários outros setores e indústrias. Tal sucesso que não existiria sem as relações públicas e as relações públicas internacionais. Por isso é tão importante analisar o que aproxima (e o que vai contribuir para aproximar ainda mais) duas áreas erroneamente consideradas distintas, como a diplomacia e as relações públicas - ou, para ser mais exato, a diplomacia de negócios e as relações públicas internacionais. Afinal, as relações públicas internacionais têm muito a ganhar com este paralelo, uma vez que poderiam ser pensadas em larga medida como um tipo de "diplomacia civil".

\section{RELAÇÕES PÚBLICAS INTERNACIONAIS}

A definição de relações públicas internacionais deve emergir tanto da análise dos termos que as compõem, quanto do efeito que tais termos impõem quando usados em conjunto. Por exemplo, o termo "relações" tem a ver com relacionamentos, isto é, o cultivo, a manutenção e o aperfeiçoamento de uma rede estratégica de contatos pessoais e institucionais. Já o termo "públicas" evoca um duplo caráter: de um lado, o contraste entre público versusprivado, ou seja, do que é feito expressamente na esfera pública; de outro lado, quando se refere aos diferentes públicos (stakeholders) que existem na sociedade. Por fim, o termo "internacional" é composto por dois outros elementos: intere nacional. De forma direta, significa 'entre nações'.

Anderson (1989) defini(u) as relações públicas internacionais como a prática de programas de comunicação distintos em vários mercados, com ações especialmente estruturadas para atender cada um deles. (...) Para Wilcox, Cameron e Xifra (2007 p. 653), as relações públicas internacionais podem ser definidas como "a atividade planejada e organizada de uma empresa, instituição ou governo, para estabelecer relações de benefício mútuo com públicos de outros países.(...). Black (1996) apresenta uma visão mais abrangente, definindo as relações públicas globais como o entendimento mútuo e que buscam suplantar as divergências geográficas, culturais e linguísticas. Mais recentemente, Wakefield (2001) descreveu as relações públicas internacionais como um processo para estabelecer e manter relacionamentos com públicos em vários países com o objetivo de minimizar ameaças potenciais para as organizações. (...) Vercic $(1996,2000,2003)$ (e) Wakefield (2001) afirma(m) que atualmente as relações públicas internacionais são praticadas de duas formas antagônicas. Na primeira, as diretrizes de relações públicas são elaboradas na matriz e aplicadas nos demais países sem nenhuma (ou quase nenhuma) adaptação às necessidades do país estrangeiro. Já na segunda, os programas de relações públicas internacionais estão totalmente desvinculados das práticas do país de origem (Ferrari, 2008, p. 22-23).

Assim, parece sensato que algumas relações possam ser de curto, médio ou longo prazos; que se originem naturalmente ou sejam propositalmente construídas; que fluam ao sabor dos acontecimentos ou sejam conscientemente capitaneadas; que visem a objetivos precisos ou que, ao contrário, careçam de intencionalidade; que permaneçam restritas aos agentes que as exercem diretamente, ou que, antes e indiretamente, envolvam ainda outros agentes; etc. Parece igualmente razoável que tais relações se deem na sociedade e que, independentemente de seu caráter público ou privado, possam ter consequências 
societais - que afetem o bem comum. Aliás, a expressão "bem comum" remete igualmente a dois outros termos de interesse: o primeiro, "bem", pode referir-se tanto à esfera econômica (uma coisa com valor e preço de mercado, submetida às regras de usufruto e propriedade) quanto à esfera social (o antônimo de mal); o segundo, "comum", evoca uma comunhão entre pares - e permite perguntar: quem e quantos são os pares, e o que comungam e em qual extensão?

\begin{abstract}
This study found that (1) business organizations and central governments were major clients of international public relations in the U.S., (2) meeting with governmental officials and congressional leaders was the primary type of activity followed by information dissemination, and (3) economic purpose led by trade promotion was the primary motive for these activities. (...) Type of international public relations activity was categorized as: (1) meeting and contact, (2) producing and disseminating information targeting general public, (3) advice and counsel, (4) governmental representation, (5) media relations and publicity, (6) event promotion and advertising, (7) research, (8) speech and creative writing, and (9) others (Lee, 2006, p. 97, 99).
\end{abstract}

De modo restrito, as relações públicas internacionais podem ser entendidas como o conjunto de relacionamentos perceptíveis na esfera pública entre nações. Contudo, tal compreensão seria limitada, pois: muitos dos relacionamentos que impactam o bem comum não são transparentes (perceptíveis) nem são conduzidos na esfera pública (não são acessíveis nem participativos); não existe apenas uma esfera pública, no singular, mas sim múltiplas esferas públicas; e, num contexto de globalização e avançadas conquistas tecnológicas, a unidade "nação" precisa reconfigurar-se (para superar as restrições políticas e geográficas). A noção de fronteiras físicas é desafiada pelas tecnologias de informação e comunicação (internet) e de transportes (imigração e turismo em massa). Por outro lado, governos e estados assistem à emergência de novos players sociais, com crescente poder e influência nos assuntos públicos.

The literature review provides the basis for developing an "in-awareness" approach to international public relations as a process of intercultural communication between client and practitioner. The pivotal question is: how do communication differences between the practitioner and client influence the communication function of public relations? (Zaharna, 2001, p. 137).

Nesse novo e mais complexo contexto, as relações públicas internacionais precisam ser repensadas para considerarem: (1) que o planejamento e a condução técnica (táticas e estratégicas) de relacionamentos sociais devem ser uma atividade expressa, formal, consentida, monitorada e responsável; (2) que o caráter público da atividade se sobrepõe a qualquer interesse privado e que o aspecto humano do indivíduo não deve ser obliterado pelo uso de terminologias coletivizantes como audiência, mercado, ou públicos; (3) que o alcance da atividade extrapola fronteiras físicas (país, estado, município) e unidades burocráticas (governo, estado) e que o conceito de "nação" torna-se fluido para significar simplesmente "pares" isto é, aqueles que se igualam (ou se consideram iguais) em alguma medida ou instância.

Perhaps a modified definition of international public relations for the 21 st century is: international public relations is the communicationcentered practice of effectively managing relationships with international audiences to foster intercultural understanding and considering cultural differences in tailoring messages to different situations, markets or publics (Hatzios, 2006, p. 84).

Logo, para que as relações públicas internacionais sejam (social, política, econômica e ecologicamente) sustentáveis, vale notar o seguinte: os relacionamentos precisam basear-se em uma mutualidade (participação equânime e justa distribuição dos benefícios); que em todos os seus processos a governança (privativa ${ }^{2}$ ) esteja submetida à accountability pública; e que 0 constante empoderamento de novos players sociais conduza à ampliação da democracia (direta e participativa) e da justiça (social e econômica). É fundamental entender que a atividade de relações públicas internacionais lida diretamente com 0 balanço de poder político e a distribuição de benefícios econômicos-e faz isso glocalmente. O fato de que serve, eventualmente, a interesses privados não é impeditivo; mas reforça a importância de manter sempre uma consideração ética mais ampla.

2 Preferiu-se o termo "privativo" em vez de "privado", pois mesmo instituições públicas, como um governo ou estado, possuem governança. e, neste caso, ficaria estranho pensar em "privado" nesse contexto. 


\section{PRINCÍPIOS DA DIPLOMACIA}

De modo geral, a diplomacia envolve três fatores fundamentais: um representante, uma indicação formal, um aceite de terceiro. 0 "diploma" é o documento que oficializa a indicação, e é um registro formal, público e transparente. Através desse ato oficial, o stakeholder A indica o stakeholder B como seu representante junto ao stakeholder $\mathrm{C}$, que 0 aceita e 0 reconhece como tal. A partir de então, o stakeholder B passa a administrar os interesses do stakeholder A de acordo com os termos prescritos no "diploma" - que funciona como uma procuração, e que normalmente declara o objeto, os limites dos poderes concedidos, além de, em alguns casos, as respectivas vedações. Dessa forma, a diplomacia é simplesmente um contrato social multistakeholder, e o nome dado ao conjunto de atividades desenvolvidas por $\mathrm{B}$, em nome de $\mathrm{A}$, junto a $\mathrm{C}$.

In a literal sense, diploma is a folded document and diplomacy encompasses the activities performed in accordance with and by those referred to in the diploma. As lucu states (2008, p. 14): "Greeks used to hand two documents[:] a recommendation letter for the proxenos called symbolia and written instructions, folded into two, called diploma". In this very general setting, diplomacy implies an appointment and a set of expected goals and behaviors (Nobre; Filimon, 2015a, p. 8).

De modo restrito, a diplomacia tem sido associada apenas a um tipo muito especial de contrato social: aquele firmado entre pessoas jurídicas do direito internacional público, a saber, reis, príncipes, nações, governos, países, ministérios, autoridades etc. Isso implicaria não apenas limitar o perfil dos stakeholders ao primeiro setor, mas igualmente circunscrever os termos passíveis de constar no "diploma" - àquilo afeito às atribuições de "funcionários públicos". Nesse sentido, os diplomatas sempre foram prepostos oficiais - que, mesmo sem serem necessariamente funcionários de carreira, sempre que investidos adquiriam o status de funcionários públicos. Isso é importante, pois um diplomata pode ser um especialista de mercado, um político de um partido, uma liderança do terceiro setor, e até um cidadão de outro país ${ }^{3}$. Não precisa pertencer, necessariamente, ao setor público.

In theory, any given agent or actor could be called diplomat whenever found formally invested as somebody else's representative and having had agreed with the terms of such delegation -which includes third partie' approvals, rules, and expectations. Put it this way, diplomacy is a multistakeholder contract for peaceful and mutual fruitful relationships - that presupposes dialogue, sympathy, negotiation, conflict management, deliberation, and decision power (Nobre; Filimon, 2015a, p. 8).

Embora o objeto constitutivo da diplomacia seja a representação, seu objetivo é, em última instância, evolutivo. Essa palavra, evolução, carrega um duplo sentido: selecionar e se desfazer do que não funciona, e selecionar e aperfeiçoar aquilo que funciona. Essa outra palavra, funcionar, quer acentuar o caráter pragmático da diplomacia (por contraste ao ideológico), mas não vai restringi-la exatamente ao "funcionalismo". Por fim, um dos pilares da diplomacia é a reciprocidade, conceito que abarca uma série importante de valores: a igualdade dos agentes, a mutualidade das relações, a equanimidade das responsabilidades. Quer dizer, os agentes têm os mesmos direitos e deveres (independentemente de seu tamanho, perfil, etc.), todos têm o mesmo poder (para falar, resolver e agir) que suas contrapartes, e qualquer benefício ou prejuízo deve ser igualmente distribuído.

Other presumptions of diplomacy assume mutual, balanced and reciprocal relationships: all sides must benefit, the benefits shall be fairly distributed, and one agent will probably echo what the counterpart has done (i.e., paying goods with goods) (Nobre, Filimon, 2015b, p. 5).

Assim, a diplomacia funciona como um coeficiente de ponderação para as diferenças dos múltiplos stakeholders envolvidos: ao mesmo tempo em que os diplomatas do stakeholder A tentam obter o máximo das relações com o stakeholderC (e vice-

3 Embora alguns países possuam legislação que restrinja estrangeiros em cargos públicos, é possível delegar a representação diplomática, em casos especiais: "um mesmo chefe de missão poderá representar dois ou mais estados (...) São casos muito raros mas que podem ocorrer (...)" (Magalhães, 2005, p. 22). 
versa), a diplomacia teoricamente impediria, via reciprocidade, a exploraçã $0^{4}$ desigual ou injusta de uns sobre os outros. Esse sistema de freios e contramedidas está lastreado no pressuposto fundamental da diversidade: os agentes se reconhecem intrinsecamente diferentes (culturas, línguas, valores etc.), fator que se comprometem mutuamente a respeitar e zelar. Em última instância, o compromisso da diplomacia não é exclusivamente o representado e seus interesses; mas, antes, todo 0 sistema em sua diversidade de stakeholders e regras que garantam seu correto funcionamento e resiliência.

\section{DIPLOMACIA E RELAÇÕES PÚBLICAS INTERNACIONAIS}

A história da diplomacia, ou, melhor, das relações consulares, registra a atuação de variados stakeholders nos negócios públicos. Por exemplo, os proxenos gregos funcionavam como diplomatas para estrangeiros (pessoas, associações ou governos) dentro da própria Grécia, ao passo que os cônsules ou aldermen desempenhavam tais funções em nações estrangeiras - embora fossem os representantes de uma corporação mercantil (no caso, a Liga Hanseática). No primeiro caso, um cidadão grego representava interesses estrangeiros dentro da Grécia; no segundo caso, um civil representava os interesses de uma entidade privada nacional em território estrangeiro. Em ambos os casos, tratava-se de diplomacia.

International public relations can serve many important functions. One of these functions is public diplomacy. Public relations scholar James Grunig (1993) argued that public diplomacy consists "essentially of the application of public relations to strategic relationships of organizations with international publics" (p. 143). Many foreign governments are looking to public relations practitioners to help them communicate their messages to their publics, including internal publics (citizens of the country) and publics of neighboring countries who are affected by a country's policies (Grunig, 1993) ([Hatzios, 2006, p. 15).

Mais recentemente, tem-se assistido à democratização da diplomacia (Nobre, 2015), isto é, à ampliação do acesso e da participação de múltiplos stakeholdersnos negócios internacionais - públicos e privados. Empresas, ONGs, celebridades e a mídia têm pressionado para pautar e formatar as políticas públicas internacionais, com foco especial para a imigração (guerra, pobreza, fome), ecologia (efeito estufa, extinção das espécies, desflorestamento), direitos humanos (mulheres, crianças, idosos, minorias religiosas), terrorismo etc. - inclusive participando diretamente, junto aos diplomatas do primeiro setor (público), de reuniões em fóruns da ONU. Essa atividade tem recebido uma série de rótulos, como: diplomacia não-governamental, diplomacia não-estatal, diplomacia cidadã, diplomacia direta, diplomacia participativa, diplomacia corporativa, diplomacia organizacional, diplomacia civil, diplomacia do terceiro setor, diplomacia de celebridades, diplomacia multistakeholder etc.

Public diplomacy as a scholarly field is built on the theoretical traditions of international relations and international communication (Gilboa, 2008; Szondi, 2008) and for communication scholars the natural host discipline is international public relations (Kunczik, 1997; Yang, Klyueva, \& Taylor, this issue). From this perspective, studies of governments' increased citizen-level diplomacy have been conducted through the lens of image-building and more recently relationship management (e.g. Fitzpatrick, 2007; Taylor, 2008). Thus, public diplomacy can be seen as a part of the successful expansion of core public relations practices such as relation building (cf. Ledingham \& Bruning, 2000) and image making (cf. Hutton, Goodman, Alexander, \& Genest, 2001) into many societal institutions as a specific mode of governance (Crouch, 2004). (Rasmussen; Merkelsen, 2012, p. 810).

Tendo em mente que as funções da diplomacia sejam a representação, a negociação, a informação (comunicação), a promoção, a proteção e a defesa dos interesses do stakeholder $\mathrm{A}$ junto ao stakeholder $\mathrm{C}$, bem como atividades efetuadas por seu representante, o stakeholder $\mathrm{B}$, de forma delegada e em caráter profissional, fica mais fácil buscar por paralelos entre a diplomacia e as relações públicas internacionais. Em primeiro lugar, ambas envolvem a indicação de representantes:

4 Explorar, aqui, vai além do sentido pejorativo; e significa beneficiar. 
na diplomacia, o diplomata; nas relações públicas internacionais, o profissional de relações públicas. Em segundo lugar, ambas trabalham essencialmente na esfera dos relacionamentos sociais. Em terceiro lugar, atuam concomitantemente na esfera local e global (glocal) - a saber, internacional, nacional, transnacional, regional etc. As novas tecnologias (internet, tv a cabo, celulares, jatos) tornaram difusos os fluxos entre o "dentro e o fora".

\begin{abstract}
PR bears strong connections to and similarities with diplomacy in a number of ways. Both are responsible for official institutional communications with other organizations and relations with wider groups or publics and are responsive to public opinion and media coverage. At a functional level, it can be argued that PR is part of the practice of diplomacy responsible for international communications and media relations as well as cultural diplomacy, which aims to enhance personal relationships between representatives of the host and target countries. It can be argued that diplomacy (political, economic, informational, cultural) is part of organizational strategic PR and that skills of diplomacy are important to effective PR. Diplomacy and PR include overt and covert aspects; entail surveillance (issues management), secrecy (confidential information, commercial and organizational secrets), and crisis management; and engage in information wars (Taylor, 1997) and psychological operations (the values of which are quite apparent in the early PR issues management literature and present in campaign planning models) (L'Etang, 2009, p. 608).
\end{abstract}

Por outro lado, para que a diplomacia e as relações públicas internacionais sigam convergindo, é preciso tomar alguns cuidados. Por exemplo: ao passo que a diplomacia se torna cada vez mais pública à medida que absorve stakeholders diversos, as relações públicas internacionais que, por definição, já são multistakeholders, devem investir ainda mais em práticas participativas, deliberativas, e democratizantes. Nesse sentido, as relações públicas internacionais têm muito que avançar quanto à reciprocidade, em seu tripé de igualdade, mutualidade e equanimidade - pois a reciprocidade é um compromisso público de respeito pelo mútuo beneficio das contrapartes. A questão do poder, se é clara para a diplomacia, é menos evidente para as relações públicas internacionais. Não que as relações governamentais e o lobby sejam estranhos à atividade de relações públicas; bem ao contrário. Todavia, as noções de "bem público" e "interesse público" têm muito futuro nas relações públicas internacionais, principalmente quando se pensa nas corporações. 0 pressuposto da diversidade em diplomacia seria igualmente promissor para as relações públicas internacionais.

\title{
DIPLOMACIA DE NEGÓCIOS
}

A palavra "negócios" pode ser associada a pelo menos duas acepções: uma mais ampla, onde negócios diz respeito a relações genéricas (em inglês, affairs); outra mais específica, onde negócios se refere a transações comerciais (em inglês, business). Daí que o Ministério das Relações Exteriores seja nomeado, em alguns países anglo-saxões, como Ministry of Foreign Affairs - e não como Ministry of International Business. Isso se deve, em parte, ao fato de que a diplomacia, para além do fator comercial, alcança mais longe, a saber: a diplomacia cultural, a diplomacia científica, a diplomacia humanitária, a diplomacia pública etc. Não há dúvidas de que a diplomacia comercial e a diplomacia econômica sejam importantes, mas não são as únicas. É uma questão de ênfase, não de exclusividade. Dito isso, vale voltar sobre a diplomacia de negócios e perguntar: o que é, afinal?

The interplay between business and diplomacy is two-fold: first, when business is brought inside the traditional diplomacy; second, when diplomacy is brought inside the traditional market. The first option approaches [the business of diplomacy] (the function of the traditional diplomatic staff) and the business' influence into diplomacy (i.e., market lobby on diplomats). The second option deals with how diplomacy can be internalized by the market (chambers of commerce activities and retired Ambassador' consultancies) and whether companies could serve as diplomatic institutions (Nobre; Filimon, 2015b, p. 7).

O primeiro passo é lembrar que a diplomacia pode ser exercida por stakeholders do primeiro setor (público), ou do segundo setor (privado), ou do terceiro setor (civil não-lucrativo), ou por membros da mídia, das universidades, dos diversos setores religiosos etc. Logo, a diplomacia de negócios pode ser entendida como um contrato social multistakeholder em duas 
esferas: a política (affairs) e a econômica (business). Em teoria, um stakeholderA elege o stakeholder B para representálo junto ao stakeholder $\mathrm{C}$, o que implica o desempenho profissional das funções diplomáticas nas esferas política e econômica. Quanto a isso, quatro observações: (1) a indicação do representante deve ser formal, pública e transparente, e pressupõe o expresso aceite das contrapartes; (2) as funções diplomáticas envolvem a execução conjunta e coerente das atividades de representação, negociação, informação (comunicação), promoção, proteção e defesa; (3) o "diploma" toma a forma de um contrato escrito, com as cláusulas que devem reger as relações profissionais e as expectativas de performance para cada uma das funções diplomáticas; (4) as esferas política e econômica são indissociáveis, mas os objetivos e as atividades para cada esfera podem constar separadamente.

\begin{abstract}
Last but not least, it is possible to say that [Business Diplomacy] encompasses the following features. First, it is based on and comes from the historical practices, researches, and studies done on the field of diplomacy. That sees it as professional, formal, technical, and scientific. Second, is private sector based: i.e., companies become diplomatic entities, either partially or entirely. Third, is supposed to service both the company and the other stakeholders. Fourth, is profit-driven and businessoriented. Fifth, is a multistakeholder social contract: so the economical goal is dependent on the political management. Sixth, it aims sustainability both as "enduring through time" and as "resilience". Seventh, is spatially global and local. Eighth, it needs a "license to operate" granted through legitimacy. Ninth, it is a peacefull set of values and behaviours, like traditional diplomacy. Tenth, it is a principled activity (Nobre; Filimon, 2015b, p. 6-7).
\end{abstract}

A diplomacia de negócios já existe e funciona amplamente em nossa sociedade, sob o guarda-chuva de uma miríade de rótulos. Por exemplo, parte ou mesmo todas as funções diplomáticas referidas são executadas cotidianamente por profissionais de comércio exterior, relações internacionais, relações públicas, por despachantes alfandegários, escritórios de advocacia, contadores, economistas, publicitários, cientistas políticos etc. Isso fica claro à medida que se analisam os componentes de cada função diplomática, a saber: representação (executivos, porta-vozes, procuradores), negociação (gerentes, consultores, relações comunitárias), comunicação (relações públicas, jornalistas, relações com a mídia), promoção (advocacy, pessoal de marketing, publicitários), proteção (planos de contingenciamento, especialistas em crise, segurança privada) e defesa (mediação, arbitragem, jurídico).

\title{
DIPLOMACIA DE NEGÓCIOS E RELAÇÕES PÚBLICAS INTERNACIONAIS
}

A melhor forma de checar os reais pontos de contato entre a diplomacia de negócios e as relações públicas internacionais é através do detalhamento de ambas as atividades. Por exemplo, Nobre e Filimon (2015b) analisaram como empresas e universidades entendiam efetivamente a diplomacia de negócios; isto é, como a definiam e explicavam em seus respectivos websites. Os autores agruparam todo este conteúdo de duas maneiras: uma, organizando-o segundo as funções da diplomacia; outra, ordenando-o de acordo com o perfil de cada atividade desenvolvida ou requerida. Todas as empresas e universidades foram registradas em uma tabela, onde cada qual recebeu uma numeração indicativa de sua posição na tabela. A seguir, os números dentro dos colchetes se referem a tais instituições e suas respectivas posições nos registros.

Representation: mediation [2, 21, 22], networking [1, 2], representatives training [6], connectivity [10], intermediation [12], corporate diplomatic corps [15], liaison [16], in your name to participate/organize events [16], connections [19], serving as your ambassador [19], interests [21], spokepersons [21].

Negotiation: operational [2], sensitization and persuasion [3], authorities [5,20], support [7, 22], in complex situations [17], new deals [21], contracts and clauses [21], mediation and arbitration [21].

Promotion: advocacy [2, 5, 21, 22], influence [1, 2, 5, 11, 22], interests [2], lobbying [7, 20], opportunities exploitation [11], coalitions [11], business [21]. 
Communication: media monitoring [1], public opinion [1], intelligence [3], optimizing [5], briefing [6, 16, 19], material developing [7], publications [9], effective channels [13], debriefing [16], events [16], media relations [16, 21], customization [19], promote open/objective conversations [21], information gathering [21], observation and reporting [21], sensitive to cultural/social/political components [21].

Defense: non-litigious dispute resolution [2, 22], courtroom and international/domestic law expertise [14], problem-solving [19], crisis management [7, 19], dispute management [21], interests [21].

Protection: interests [7], foresighting [7], risks anticipation [12], vulnerability to risks [11], secure business relation [13, 16], prevent/ solve/minimize conflicts [13, 21] and misunderstandings [16], identify the tricks [16].

Tomem-se, por exemplo, as atividades agrupadas na categoria "comunicação": monitoramento da mídia; opinião pública; inteligência; otimização; briefing; desenvolvimento de material; publicações; canais efetivos; debriefing; eventos; relações com a mídia; personalização; promover conversações abertas e objetivas; reunião de informação; observar e reportar; sensibilidade a componentes culturais, sociais e políticos. Muitos desses (senão todos) fazem parte das atribuições do profissional de relações públicas internacionais. Mas há, ainda, outras atividades obviamente relacionadas às atribuições do profissional de relações públicas internacionais, a saber: networking, conectividade, ligações, intermediação, sensibilização, persuasão, apoio, organização e participação de eventos, atividades de porta-voz, mediação, advocacy, coalisões, relações pacíficas, gerenciamento de crise, resolução de problemas, antecipação de riscos, prevenção e minimização de conflitos e de mal-entendidos etc. A segunda forma de elencar as atividades e subatividades também é registrada a seguir.

Relationships cultivation: ecosystem of actors and engagement of parties [1], privileged contacts [3], opinion leaders [4], B2B contacts [5], networks building [7,11], network cartography [12], broker [10], key actors [12], government [13,14], critical relationships leveraging [14], right person to address [16], identify and approach of contacts [16], opinion-makers [18], source local partners [21].

Decision makers/making: access [2], identify and qualify [3, 4], shaping [5], highest levels lobbying [7], optimizing [12], patterns [12].

Analytical skills: in-depth knowledge [2], deciphering [7, 12], research [9, 10], auditing [9], risk exposure [12], publicized and published material [21].

Synthetic skills: advising [2, 7, 10, 19, 22], counselling [3], consulting [5, 6, 9, 17, 20, 21], complex international problems solving [14].

Support to business. set the stage [10], settlement [6, 21, 22], help to start [3], development [2], trade and investment [5], staff relocation [6], projects [7], political [7], training [9, 21], to assist with legal/policy needs [15], tender preparation [16], meeting coordination [16], event logistics [16], secure position in foreign markets [17], identify opportunities [21].

Strategy. designing [3, 7], briefing [6], crisis management [7], geo-strategy [16], and tactic [19].

Internationalization: emerging countries [3], new markets penetration abroad [7].

Monitoring: market conquest [3], trends [7], crisis and rupture anticipation [12], reputation [12], tender [16], local authorities [17].

Principled: contracts [2], etiquette [6], law [14], international/domestic regulations [14], protocol [21].

Bridging: cultures [6], local culture and socio economic dynamics [13, 16], interpretation [16], customizing your communications to local circumstances [19], cross-cultural translation of ideas / beliefs / attitudes / behaviours [21].

Bonding: commercial engagements [10], coalitions [11], potential partnerships [12], shared views [16], secure partnership [17], government engagement [18], specific associations [19], making overseas introductions [19], engaging accredited intermediaries [21], cooperation [21].

Leading. in-depth guidance [4], mentoring [6], open the right door and say the right thing to the right audience abroad [15], sending the right signals [16].

Legitimacy. reputation [7, 9, 11, 12, 19], social capital [9], credibility [10], make the right impression [10].

Multistakeholders: continuously changing relationships with [9], dynamic and complex interactions with [11], significant experience working with [14], secure / efficient / profitable relationship with [16], stakeholder networks [19].

Glocal: different structured and informal fora [9], local information [13], experience in a large number of countries throughout the world and in domestic institutions [14], corporate expansion across the globe [15], local media attention [16]. 
Intelligence: knowledge [10], market intelligence [12], local value added information [13, 16], legal expertise [14], environment and issues understanding [16], inside expertise [19].

Sustainability. long term stability [13], long term relation [16], sustainable partnership [17], sustainable strategic network [21].

Peaceful: create a smooth path [20], promote constructive conversations [21], creating a favourable climate [22].

Goal: success [13, 15], profitable business relation $[13,16]$, save cost and time $[13,16]$.

Percebe-se mais claramente que algumas dessas atividades ou expertises pertencem ao rol de atribuições do profissional de relações públicas internacionais, a ver: o cultivo de relacionamentos; a participação nos processos decisórios; habilidades analíticas e sintéticas (auditoria de riscos, aconselhamento); apoio a negócios; olhar e agir estratégico; internacionalização; monitoramento (midiático e de reputação); atividade responsável (com princípios éticos, etiqueta, protocolo); construção de pontes (culturais, sociais, políticas); desenvolvimento de laços (comerciais, parcerias, cooperações); liderança; cultivo de legitimidade (licença para operar); multistakeholder, glocal; atividade de inteligência (informação, conhecimento, posicionamento estratégico); sustentabilidade e resiliência; pacifismo; e orientada a resultados e metas.

\section{CONSIDERAÇÕES FINAIS}

Muito embora não seja acurado defender a perfeita sinonímia entre diplomacia e relações públicas, ou entre diplomacia de negócios e relações públicas internacionais, é, sim, cabível afirmar que ambas comungam, sem embargo, mais afinidades do que diferenças. Isso se deve, em grande parte, ao fato de que sejam intensamente dependentes da comunicação e dos relacionamentos; de que estejam enraizadas no processo de globalização e consequentes fluxos (de pessoas, de produtos etc.); e de que naveguem, ao mesmo tempo, na esfera política (affairs) e na esfera econômica (business).

Claro, outros fatores também contribuem para aproximá-las, como o de que pertencem ambas ao setor de prestação de serviços, de que estejam imbrincadas com as atividades afetadas pelo avanço das novas tecnologias de informação e comunicação (mídias e imprensa) e de transportes (viagens, turismo, imigração), de que trabalhem ambas com a opinião pública e com a construção de um capital (social, simbólico, reputacional), dentre outros.

Todavia, há indícios de que igualmente se diferenciem. Por exemplo, na questão da reciprocidade (um pilar na diplomacia, mas um ponto a ser desenvolvido nas relações públicas internacionais), da diversidade (outro pilar da diplomacia, mas ainda de incipiente tratamento pelas relações públicas internacionais), e, por que não dizer?, a questão do "bem público" e do "interesse público" (a exigir a reconsideração da importância de processos mais participativos e deliberativos dentro das relações públicas internacionais).

\section{REFERÊNCIAS}

FERRARI, Maria Aparecida. A prática das relações públicas internacionais na sociedade contemporânea. Anuário Unesco/ Metodista de Comunicação Regional, a. 12, n. 12, p. 15-29, 2008.

HATZIOS, Adamantia Kriton. Perceptions of utility and importance of international public relations education among educators and practitioners. Master Thesis, University of Georgia, Athens, Georgia, 2006. 
L'ETANG, Jacquie. Public Relations and diplomacy in a globalized world: an issue of public communication. American Behavioral Scientist, v. 53, n. 4, p. 607-626, 2009.

LEE, Suman. An analysis of other countries' international public relations in the U.S. Public Relations Review, n. 32, p. 97-103, 2006.

MAGALHÃES, José Calvet de. Manual diplomático: direito diplomático e prática diplomática. Lisboa, Editorial Bizâncio, 2005.

NOBRE, Guilherme Fráguas. A democratização da diplomacia: novos atores na oferta e demanda por serviços diplomáticos. Orbis Latina, v. 5, n. 1, p. 7-18, 2015.

NOBRE, Guilherme Fráguas; FILIMON, Nela. Business diplomacy in a consulariate format: global legitimacy challenges for an industry to be. In: INTERNATIONAL BUSINESS DIPLOMACY CONFERENCE, 1st, Zwolle, Netherlands, december 4th, 2015. Proceedings... Zwolle: Windesheim University of Applied Sciences, 2015a.

. What companies and universities mean by business diplomacy: a global legitimacy challenge. In: INTERNATIONAL BUSINESS DIPLOMACY CONFERENCE, 1st, Zwolle, Netherlands, december 4th, 2015. Proceedings... Zwolle: Windesheim University of Applied Sciences, 2015b.

RASMUSSEN, Rasmus Kjærgaard; MERKELSEN, Henrik. The new PR of states: how nation branding practices affect the security function of public diplomacy. Public Relations Review, n. 38, p. 810-818, 2012.

ZAHARNA, Rhonda S. "In-awareness" approach to international public relations. Public Relations Review, n. 27, p. 135-148, 2001.

Artigo recebido em 04.06.2016 e aprovado em 20.06.2016. 